\title{
Aneurysmal bone cyst of the mandible, mimicking follicular cyst
}

\author{
Zornitsa Mihaylova, Pavel Stanimirov, \\ Evgeniy Aleksiev
}

Dept. of Dental, Oral and Maxillofacial Surgery, Faculty of Dental Medicine, Medical University - Sofia; Maxillofacial Surgery Clinic, Alexandrovska Hospital;

\begin{abstract}
Aneurysmal bone cyst $(A B C)$ is a rare benign, non-odontogenic osteolytic lesion of the jawbones. Commonly the clinical presentation consists of pain, significant bone expansion and facial asymmetry. We report a case of a 14-year-old asymptomatic patient, with aneurysmal bone cyst, identified on a routine $X$-ray, mistaken with dentigerous cyst. Thorough surgical removal of the cystic lesion was carried out, followed by histopathological examination confirming the diagnosis. Long term follow-up showed no complications or recurrence.
\end{abstract}

Keywords: aneurysmal bone cyst, osteolytic lesions, bone cysts, non-odontogenic cysts

\section{Introduction}

Aneurysmal bone cyst $(A B C)$ is a relatively rare non-neoplastic osteolytic lesion. Significant bone expansion is commonly observed. It consists of varying in size serum- or blood-filled spaces separated by trabeculae. The spaces may contain osteoid tissue and osteoclast-like giant cells (1). ABC has been first described in 1893 by Van Arsdale as an ossifying hematoma (2), while the first case identified in the jaws has been reported by Bernier and Bhaskar in 1958 (3).

ABCs primarily affect the long bones and vertebrae, with only $1 \%$ to $3 \%$ incidence in the jaws (3). The mandible is more commonly affected than the maxilla, with a ratio of $2: 1$, usually found in the posterior 
region (4); Urs et al. (5) reported a ratio of presentation of the lesion in male to female and in maxilla to mandible of $3: 6$ and 1:6, respectively.

According to reports in the literature, the clinical presentation of $A B C$ s might be quite variable, from slowgrowing asymptomatic appearance to cysts with highly aggressive and destructive manifestation $(6,7)$. Cases of giant $\mathrm{ABCs}$ with multicentric radiographic appearance, associated with significant functional disturbances have been reported (8). Pain and swelling are found the most common presenting features (5).

Our aim is to report a clinical case of patient with aneurysmal bone cyst in the left posterior mandible, mimicking the clinical presentation of odontogenic cyst.

\section{Case report}

We report a clinical case of a 14-year-old male patient referred to the Maxillofacial surgery clinic, with a 1year history of osteolytic lesion in the distal aspect of the mandibular left wisdom tooth. The lesion has been taken for dentigerous cyst and surgical removal of the wisdom tooth has been recommended. However, due to the lack of symptoms, the patient does not seek additional dental consultations and treatment since then.

At the time of the presentation in our clinic, the patient does not have complaints of pain, functional or sensitivity disturbances and facial asymmetry. No history of trauma or surgery in the maxillofacial area was reported. We see no evidence of swelling, suppuration or any signs of infection during the intra- and extraoral examination. The patient had normal range of mandibular motions in all directions.

Cone beam computed tomography (СВCT) was performed, revealing the presence of $12 \mathrm{~mm} / 13,5 \mathrm{~mm}$ in size well defined, unilocular osteolytic lesion, distally to the crown of the unerupted third mandibular molar (tooth germ) on the left mandible. The upper wall of the mandibular canal was found to be interrupted due to the bone resorption, with $12 \mathrm{~mm}$ „window“. No bone deformity or expansion of the jaw was observed (Fig.1A,1B).

Surgical treatment was performed through intraoral approach under local anesthesia. After raising a mucoperiosteal flap, the tooth germ was removed, followed by an appropriate curettage of the bone cavity. Prior to removal, the cystic-like lesion was filled with brown fluid and its wall was found to be thin and friable. Histopathological examination revealed the presence of unclothed blood, woven bone and trabeculae of osteoid, as well as giant multinucleated cells. The results of all, the clinical and imaging findings, together with the histopathological examination confirmed the diagnosis of ABC. Long-term follow up of our patient showed lack of early or late postoperative complications and morbidity.

\section{Discussion}

$A B C$ is a benign non-neoplastic pathological finding. The term "aneurysmal" has been introduced by Jaffé and Lichtenstein in 1942 (9) due to the significant bone expansion usually observed. ABC in the jawbones is not commonly found. 
$A B C$ is usually identified in the first decades of life, with peak between the age of 10 and 20 years (10) and lack of convenient reports in the literature about significant sex predilection. The etiology and pathogenesis are still debated. Several cases of $A B C$ associated with pregnancy have been reported in the literature, suggesting potential relation to hormonal disturbances (8).

According to the update from the 4th Edition of the World Health Organization Classification of Head and Neck Tumors (11), ABC is classified as a giant cell lesion of the jawbones, due to its strong connection to particular genetic alterations. The location of the cyst is intramedullar or juxtacortical (also called subperiosteal), as it is reported to be related to the causative factor (12). The subperiosteal ABC is considered to be of traumatic origin, while the intramedullar presentation is associated with a preexisting lesion. It appears in bones with an abundant bone marrow, high venous pressure and venous occlusion. Therefore, it is more commonly seen in maxillofacial bones when compared to skull bones.

Three variants of $A B C$ have been described in the literature (12): 1.conventional or vascular type with more aggressive and rapid growth, 2.solid type with slow growth and discrete clinical appearance and 3.mixed type. According to the clinical and radiographic presentation, three stages have been distinguished (13): 1st stage - inactive, with well-defined periosteal and sclerotic borders, 2nd stage - active, with well-defined radiographic appearance and interrupted periosteal border, 3rd stage - aggressive, with rapid growth and diffuse ill-defined radiographic appearance. In the first stage, there might be lack of complaints and lack of clinical evidence for bone pathology, found on extra- or intraoral examination, as the ABC is recognized only radiographically.

Wide range of pathologies with osteolytic radiographic appearance is included in the differential diagnosis of $A B C$, i.e. odontogenic cysts, non-odontogenic jawbone cysts, ameloblastoma, ossifying fibroma, fibrous dysplasia, giant-cell granulomas, osteosarcoma, etc. They all have to be considered preoperatively in order to avoid mistakes in diagnosis and treatment plan.

In the present case, the cystic formation could be easily mistaken with odontogenic jawbone cyst (dentigerous cysts), due to its location in the distal aspect of the mandibular left wisdom tooth. Furthermore, there is no medical history of pain, swelling or any functional disturbances. Absence of pain symptoms and the blood-filled cavity are considered unusual clinical findings and are reported in very few cases $(14,15)$. Therefore, the location of the cyst in our case, together with the medical history may lead to inaccurate diagnosis and insufficient surgical management. Removal of the tooth together with the lesion should be considered. Based on the thin and friable cystic wall that was found during the surgical enucleation, we assumed the presence of non-odontogenic lesion. The results of the histopathological examination confirmed the diagnosis of $A B C$.

The treatment plan must be in accordance with the location of the $A B C$, clinical presentation and radiographic findings. As in all benign bone lesions, non-aggressive inactive cysts could be removed by enucleation and curettage, while symptomatic rapidly-growing cysts require resection, followed by bone graft or metal plate reconstruction. In the presented case, a thorough curettage was performed after the extraction of the tooth and the cyst removal. Long term follow-up showed no recurrence and no functional complications in the early and late postoperative period. 

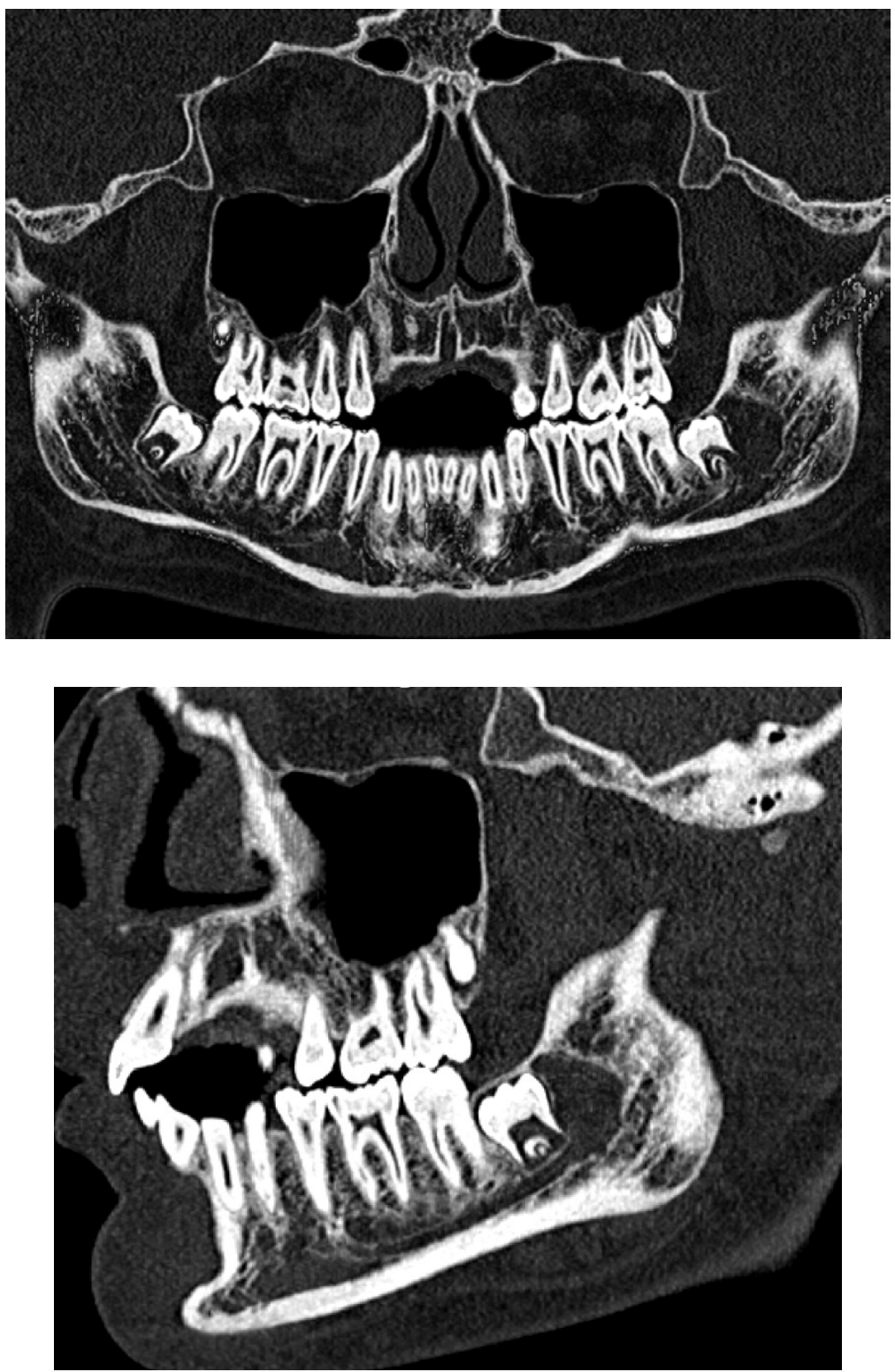

FIGURE LEGENDS:

Fig. 1 A,B - Preoperative computed tomography, showing the presence of unilocular osteolytic lesion in the distal aspect of the left mandibular wisdom

\section{Conclusion}

$A B C$ in the jawbones is a relatively uncommon clinical finding. Bone expansion and fascial asymmetry are commonly observed. However, cases of much more discrete appearance, mimicking odontogenic cysts of the jaws could be observed. Prompt and adequate surgical removal of the lesion is associated with excellent long term prognosis. 


\section{References}

1. Schajowicz F: Histological typing of bone tumors. World Health Organization International Histological Classification of Tumors. Berlin: Springer Verlag,1993.

2. Gadre KS, Zubairy RA. Aneurysmal bone cyst of the mandibular condyle: Report of a case. J Oral Maxillofac Surg 2000;58:439-43.

3. Bernier J, Bhaskar S: Aneurysmal bone cyst of the mandible. Oral Surg Oral Med Oral Pathol 1958;11:1018-28.

4. Sun ZJ, Sun HL, Yang RL, et al. Aneurysmal bone cysts of the jaws. Int J Surg Pathol 2009;17:31122.

5. Urs $\mathrm{AB}$, Augustine $\mathrm{J}$, Chawla $\mathrm{H}$. Aneurysmal bone cyst of the jaws: clinicopathological study. J Maxillofac Oral Surg 2014;13(4): 458-463.

6. Motamedi M, Khodayari A. Aneurysmal bone cyst mimicking a malignancy. J Oral Maxillofac Surg 1993;51:691-5.

7. Trent C, Byl F. Aneurysmal bone cyst of the mandible. Ann Otol Rhinol Laryngol 1993;102:91724.

8. Hnenny L, Roundy N, Zherebitskiy V, Grafe M, Mansoor A, Dogan A. Giant aneurysmal bone cyst of the anterior cranial fossa and paranasal sinuses presenting in pregnancy: case report and literature review. J Neurol Surg Rep 2015; 76(02): e216-e221.

9. Jaffe HL, Lichtenstein L. Solitary unicameral bone cyst with emphasis on the roentgen picture, the pathologic appearance and the pathogenesis. Arch Surg 1942;44:1004-25.

10. Struthers PJ, Shear M. Aneurysmal bone cyst of the jaws. (I). Clinicopathological features. Int J Oral Surg 1984;13:85-91.

11. Wright JM, Vered M. Update from the 4th edition of the World Health Organization classification of head and neck tumours: odontogenic and maxillofacial bone tumors. Head Neck Pathol 2017;11(1): 68-77.

12. Shear M, Speight PM. Aneurysmal bone cysts. In: Shear M, Speight PM, eds. Cysts of the Oral and Maxillofacial Regions. Oxford: Blackwell Publishing, 2007:150-5.

13. Capanna R, Betelli G, Biagini R, et al. Aneurysmal bone cyst of long bones. Ital J Orthop Traumatol 1985;11:409-17.

14. Motamedi MH, Behroozian A, Azizi T, Nazhvani AD, Motahary P, Lotfi A. Assessment of 120 maxillofacial aneurysmal bone cysts: a nationwide quest to understand this enigma. $J$ Oral Maxillofac Surg 2014;72:1523-30.

15. Flores IL, Hamilton ME, Elaine-de-Fátima Zanchin-Baldissera A, Uchoa-Vasconcelos C, et al. Simple and aneurysmal bone cyst: aspects of jaw pseudocysts based on an experience of Brazilian pathology service during 53 years. Med Oral Patol Oral Cir Bucal 2017;22(1): e64.

\section{Corresponding author:}

Zornitsa Mihaylova, DDS, PhD; Faculty of Dental medicine,

1 G. Sofiyski str., Sofia, Bulgaria

Email: dr.z.mihaylova@gmail.com

Tel: :+359882492209 